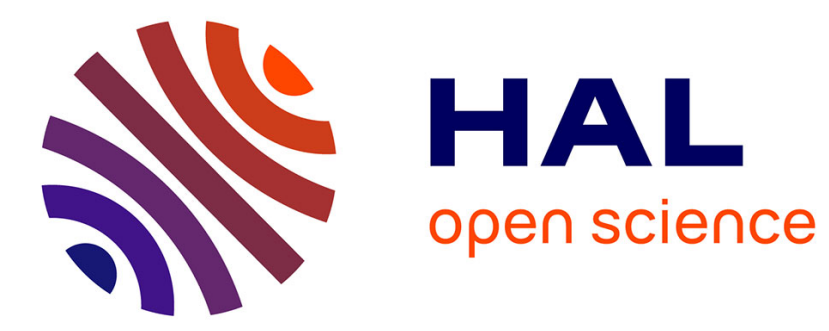

\title{
Transfer-matrix modeling of four-wave mixing at the band edge of a one-dimensional photonic crystal
}

\author{
Philippe Delaye, Magali Astic, Robert Frey, Gérald Roosen
}

\section{To cite this version:}

Philippe Delaye, Magali Astic, Robert Frey, Gérald Roosen. Transfer-matrix modeling of four-wave mixing at the band edge of a one-dimensional photonic crystal. Journal of the Optical Society of America B, 2005, 22 (11), pp.2494-2504. hal-00671116

\section{HAL Id: hal-00671116 https://hal-iogs.archives-ouvertes.fr/hal-00671116}

Submitted on 16 Feb 2012

HAL is a multi-disciplinary open access archive for the deposit and dissemination of scientific research documents, whether they are published or not. The documents may come from teaching and research institutions in France or abroad, or from public or private research centers.
L'archive ouverte pluridisciplinaire HAL, est destinée au dépôt et à la diffusion de documents scientifiques de niveau recherche, publiés ou non, émanant des établissements d'enseignement et de recherche français ou étrangers, des laboratoires publics ou privés. 


\title{
Transfer-matrix modeling of four-wave mixing at the band edge of a one-dimensional photonic crystal
}

\author{
Philippe Delaye, Magali Astic, Robert Frey, and Gérald Roosen \\ Laboratoire Charles Fabry de l'Institut d'Optique, du Centre National de la Recherche Scientifique, \\ de l'Université Paris Sud, Centre Scientifique Paris Sud, Bât. 503, 91403 Orsay cedex, France
}

Received March 8, 2005; revised manuscript received May 19, 2005; accepted May 27, 2005

\begin{abstract}
We present a modeling of a degenerate four-wave-mixing nonlinear process in one-dimensional photonic crystals. The model is based on the nonlinear extension of the transfer-matrix description of propagation in the structure. The influence of light localization, near the band edge of the structure, on the enhancement of the phase-conjugate reflectivity is studied. The phase-conjugate reflectivity is shown to increase as the eighth power of the number of layers with an additional large dependence on the index contrast of the structure. In both cases the enhancement is accompanied by a strong reduction of the resonance width, which may lead to some limitation of the enhancement when ultrashort pulses are used. A strong influence of the losses on the nonlinear efficiency of the structure is also predicted with a great importance of scattering losses at the multiple interfaces of the structure. (c) 2005 Optical Society of America
\end{abstract}

OCIS codes: $190.4380,230.3990$.

\section{INTRODUCTION}

Several functions have been successfully inserted in photonic crystals, making such devices potentially interesting for optical microcircuits, ${ }^{1,2}$ provided nonlinear functions can also be implemented on the same device. This evidently requires the existence of very efficient thirdorder optical nonlinearities on very short interaction lengths operating at low optical power. One way to obtain those efficient functions is to use the light localization properties of photonic crystals linked to the group-velocity reduction near a band extremum. This enables a large increase of light intensity inside the structure, which is particularly interesting for nonlinear effects. ${ }^{3-8}$ Future development of these nonlinear functions will require the understanding of the interactions between the nonlinearity and the structure of the material, for the optimization of the photonic-crystal parameters. The development of analytical models such as the one presented here will enable this comprehension and optimization.

The context of our work is the study of the influence of the material structuring on the efficiency of the thirdorder nonlinearities. We focus on the third-order nonlinearities for their ability to realize functions for all-optical processing through bistability phenomenon, Kerr-phase change, or four-wave-mixing phase conjugation and parametric conversion. ${ }^{9,10}$ The influence of material structuring on the third-order nonlinearities is very important. The latter depends on the fourth power of the local intensity in the material, which will lead to a large exaltation of the nonlinearity of the structures, even with relatively low localization of light. ${ }^{11}$

Among the different nonlinear interactions, we choose to consider degenerate four-wave mixing, which is of particular interest, as it presents the advantage of being sensitive to the decrease of the group velocity or the enhance- ment of light localization only, without any phasematching problem, which is present, for example, in second-harmonic-generation experiments. ${ }^{4,12,13}$ In the four-wave-mixing process a phase-conjugate beam is created $^{14}$ that is counterpropagating to the signal beam. The value of the phase-conjugate reflectivity, defined as the ratio of the output conjugate and input signal intensities, gives a direct evaluation of the nonlinear efficiency of the structure. For the sake of simplicity, the four-wavemixing phenomenon is the only nonlinear phenomenon considered in our study. Influence of other nonlinear effects, such as bistability due to Kerr effect, which may occur at high intensities, is then neglected. This lowintensity condition can be easily applied experimentally and theoretically, leading to a simplification of the modeling and an easier interpretation of the numerical calculation while keeping the main features of the four-wavemixing process unaltered. This low-intensity regime considered in our analysis has another consequence regarding the approximations made for modeling, especially the slowly varying envelope approximation (SVEA), ${ }^{14}$ which will be fulfilled contrarily to other nonlinear phenomenon such as bistability, for which validity is not necessarily gained in photonic crystals. ${ }^{15}$

Section 1 presents the theoretical modeling of fourwave mixing in a $1 \mathrm{D}$ photonic crystal, using the transfermatrix modeling that is extended to include the nonlinear four-wave-mixing interaction between the beams. Linear and nonlinear matrices are calculated in each layer of the structure, a simple product of these matrix giving both the linear and phase-conjugate reflectivity of the structure. In Section 2, these matrices are also used to calculate the influence of the parameters of the structure (number of layers, index contrast,...) and of the experiment (signal beam incidence angle, wavelength,...) on the 


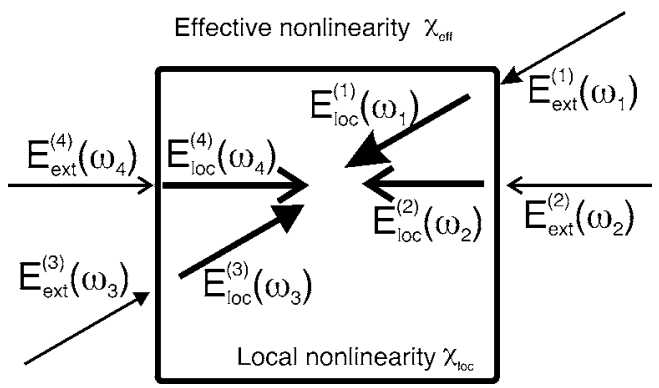

Fig. 1. Schematic principle of four-wave-mixing process.

localization of light and the amplitude of the phaseconjugate reflectivity, with this study giving hints for the optimization of the structure. Finally, Section 3 shows the influence of losses (both scattering at the interface and material absorption in the layers) on the phase-conjugate reflectivity, showing the detrimental effect of light localization that exacerbates those losses and giving some values of the maximum acceptable losses the photonic crystal could have.

\section{THEORY OF FOUR-WAVE MIXING IN A 1D PHOTONIC CRYSTAL}

Four-wave mixing is a very useful nonlinear process used for the characterization of third-order nonlinear materials. ${ }^{14}$ In this phenomenon (Fig. 1) two counterpropagating pump beams interact in the nonlinear material with a signal beam to create a so-called phaseconjugate-reflected beam that propagates contradirectionally to the signal beam. If all the beams have the same wavelength, we are in a degenerate configuration and the phase-matching condition is automatically fulfilled, whatever the wavelength of the beams is. As a consequence, the measurement of all the beam intensities allows a direct and easy determination of the thirdorder nonlinear susceptibility. Hereafter, we show that light localization can greatly enhance the optical nonlinearities and quantitatively express the intensities of the output signal and conjugate intensities in the parametric (or low-pump-depletion) approximation in 1D photonic crystals.

\section{A. Light Localization and Effective Nonlinear Susceptibilities}

In any nonlinear process, the amplitudes of the fields interacting in the nonlinear medium are those actually existing inside the material. On the other hand, the efficiency of the nonlinear process is calculated using the intensities of the different waves outside the medium (Fig. 1). As a consequence, for the third-order nonlinear processes considered here, the nonlinear polarization is expressed as ${ }^{14}$

$$
\underline{P_{\mathrm{NL}}^{(3)}}\left(\omega_{4}\right)=\underline{\underline{\underline{\chi_{\mathrm{eff}}^{(3)}} E_{\mathrm{ext}}^{(1)}}}\left(\omega_{1}\right) \underline{E_{\mathrm{ext}}^{(2)}}\left(\omega_{2}\right) \underline{E_{\mathrm{ext}}^{(3)}}\left(\omega_{3}\right),
$$

using the outside field amplitude $\underline{E_{\text {ext }}^{(\mathrm{j})}}\left(\omega_{j}\right)(j=1,3)$ and the effective nonlinearity $\underline{\underline{\chi_{\text {eff }}^{(3)}}}$ of the effective medium, consid-

ered as homogenous even if it is structured, or as

$$
\underline{P}_{\mathrm{NL}}^{(3)}\left(\omega_{4}\right)=\underline{\underline{\chi_{\mathrm{loc}}^{(3)} E_{\mathrm{loc}}^{(1)}}\left(\omega_{1}\right)} \underline{E_{\mathrm{loc}}^{(2)}}\left(\omega_{2}\right) E_{\mathrm{loc}}^{(3)}\left(\omega_{3}\right),
$$

when the local field amplitude $E_{\text {loc }}^{(\mathrm{j})}\left(\omega_{j}\right)(j=1,3)$ and the local intrinsic third-order nonlinear susceptibility tensor $\underline{\underline{\chi_{\text {loc }}^{(3)}}}$ are used.

This imposes to relate the internal (or local) fields really present in the material and creating the effect, to the external fields really measured, using the relation

$$
\underline{E_{\text {loc }}^{(\mathrm{j})}}\left(r, \omega_{j}\right)=f_{j}\left(r, \omega_{j}\right) \underline{E_{\text {ext }}^{(\mathrm{j})}}\left(r, \omega_{\mathrm{j}}\right),
$$

where $f_{j}\left(r, \omega_{j}\right)$ is the local field factor of the field $j$ at the position $r$ for frequency $\omega_{j}{ }^{16}$

The expressions giving the linear $\chi_{\text {eff }}^{(1)}\left(r, \omega_{4}\right)$ and thirdorder nonlinear $\underline{\underline{\chi_{\text {eff }}^{(3)}}}\left(r, \omega_{1}, \omega_{2}, \omega_{3}\right)$ effective susceptibility

tensors are then related to the corresponding local susceptibility tensors $\underline{\underline{\chi_{\text {loc }}^{(1)}}}\left(r, \omega_{4}\right)$ and $\underline{\underline{\chi_{\text {loc }}^{(3)}}}\left(r, \omega_{1}, \omega_{2}, \omega_{3}\right)$ by $^{11,16,17}$ :

$$
\begin{gathered}
\underline{\underline{\chi_{\mathrm{eff}}^{(1)}}}\left(r, \omega_{4}\right)=f_{4}\left(r, \omega_{4}\right) \underline{\underline{\underline{\chi_{\mathrm{loc}}^{(1)}}}}\left(r, \omega_{4}\right), \\
\underline{\underline{\underline{\underline{\mathrm{Z}}}}}\left(r, \omega_{1}, \omega_{2}, \omega_{3}\right)=f_{4}\left(r, \omega_{4}\right) f_{1}\left(r, \omega_{1}\right) f_{2}\left(r, \omega_{2}\right) f_{3}\left(r, \omega_{3}\right) \\
\times \\
\times \underline{\underline{\chi_{\text {loc }}^{(3)}}}\left(r, \omega_{1}, \omega_{2}, \omega_{3}\right) .
\end{gathered}
$$

Equation (4a) shows that a wave of frequency $\omega_{j}$ and polarization $\hat{\mathbf{e}}_{j}$ propagating in a material where localization occurs [i.e. where $f_{j}\left(r, \omega_{j}\right)$ is greater than 1] sees a greater effective refractive index $n_{\mathrm{eff}}\left(r, \omega_{j}\right)=\left\{1+4 \pi\left[\hat{\mathbf{e}}_{j} \cdot \underline{\underline{\chi_{\mathrm{eff}}}}\right.\right.$ $\left.\left.\left(r, \omega_{j}\right) \hat{\mathbf{e}}_{j}\right]\right\}^{1 / 2}$ than a medium without localization having $\overline{\overline{\text { an }}}$ identical local refractive index. Thus the light localization is directly related to the slowing down of the light wave propagating in the medium. ${ }^{18}$

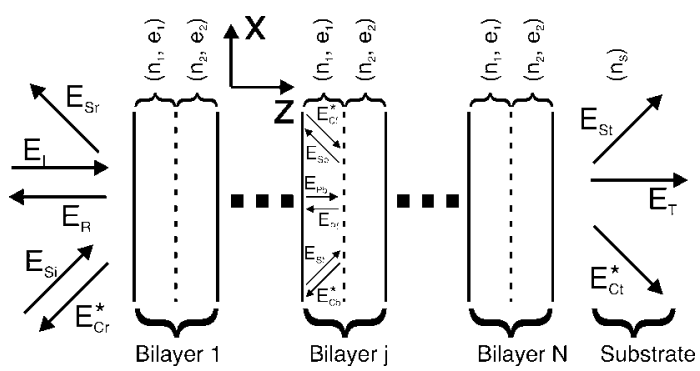

Fig. 2. Structure of the 1D photonic crystal for four-wavemixing calculations. The multilayer structure is surrounded by air of index $n_{0}=1$ at the entrance and by the substrate of index $n_{S}$ at the output. 
Moreover, as shown by Eq. (4b), the localization of light is essential for the efficiency of third-order nonlinear processes, as the effective nonlinear susceptibility increases as the fourth power of the local field factor (supposing here an identical factor for all the waves). In fact, more generally, the $n$ th-order effective nonlinear susceptibility varies as the $(n+1)$ th power of the local field factor. ${ }^{11,16} \mathrm{~A}$ localization factor as low as $f_{j}\left(r, \omega_{j}\right)=3.3$ induces an effective nonlinearity a hundred times greater than in the same material without light localization. The gain in effective nonlinear susceptibility is even a thousand times for a localization factor of only 5.6. This light localization might have a different origin, either of microscopic origin linked to the atoms of the nonlinear media or macroscopic origin owing to the structuring of the material at the scale of the wavelength. In bulk material, the local field effect is due only to the localization of electrons near the nucleus [the local field factor $f_{j}\left(r, \omega_{j}\right)$ is thus maximal for ionic compounds and equal to 1 for metals]. ${ }^{16}$ In subsection 2.B, we will suppose that this effect is already incorporated in the value of the local linear and nonlinear susceptibilities of the "bulk" material used in the structures and will consider only the effect of the localization of light due to the structuring of the nonlinear material.

B. Degenerate Four-Wave Mixing in Structured Materials In this section the phenomenon of degenerate four-wave mixing ${ }^{14,17}$ is modeled in a structured material. This material is a $1 \mathrm{D}$ photonic crystal, i.e., a stack of $N$ bilayers made of two different nonlinear materials of different refractive indices and thicknesses (Fig. 2). Basically, the layers have quarter-wavelength thicknesses, but this is not a constraint in the modeling and those thicknesses can be varied without any problem throughout the structure (with the consequence that the loss of periodicity prevents us from finding a general analytical expression for the transmission matrix as in previous models ${ }^{18}$ ).

Concerning the geometry of the beams of the fourwave-mixing process in our device, only two beams (forward pump and signal beams) are incident on the structure. The forward-pump beam is sent on the photonic crystal with normal incidence, and the backward-pump beam is created by its reflection on the structure (and, in main cases, on a mirror placed on the back side of the photonic crystal). The signal beam, possibly incident at a different angle, interacts with the pump beams to create the conjugate beam. It is also reflected on the structure, as is the reflected signal beam, which also interacts with the pump beams to create a conjugate beam that is identical to the other one after it reflects on the structure. This means that in fact six waves interact in the photonic crystal through two four-wave-mixing processes: the forward $\left(E_{\mathrm{Pf}}\right)$ and backward $\left(E_{\mathrm{Pb}}\right)$ pump waves, the forward $\left(E_{\mathrm{Sf}}\right)$ and backward $\left(E_{\mathrm{Sb}}\right)$ signal waves, and the forward $\left(E_{\mathrm{Cf}}\right)$ and backward $\left(E_{\mathrm{Cb}}\right)$ conjugate waves (Fig. 2).

The basis of the modeling is a transfer-matrix calculation well known to model the linear transmission and reflectivity of such structures. ${ }^{18-20}$ This model is generalized here to the case of the nonlinear propagation of the signal and conjugate beams using the pump local-field factor also derived from the calculation of the linear propagation of the pump beams in the structure.

\section{Linear Propagation of the Pump Beam}

For the sake of simplicity the parametric regime is assumed for the four-wave-mixing process, i.e., no change of pump-beam intensities occurs owing to energy transfer from the pump beams toward the signal and conjugate beams. In the same manner pump changes induced by nonlinear effects (an optical Kerr effect or two-photon absorption) are neglected as well. This approximation corresponds to a regime of sufficiently low intensity and small nonlinear effects; although the four-wave-mixing process at high power is actually strongly influenced by these effects $^{8}$, these approximations do not hamper the validity of the results presented hereafter, since our study is devoted mainly to delineating the influence of the structuring of the material on the effective third nonlinearity.

The transfer-matrix model ${ }^{19,20}$ allows us to calculate the pump-beam intensities (forward and backward) at the entrance of each layer i.e., at position $r_{n_{i}}^{(j)}=0$, (at the entrance of the $j$ th media of index $n_{i}$ ) taken after the interface by backward propagation of matrix produces from the output

$$
\begin{aligned}
\underline{E_{\mathrm{Pin}}}\left(r_{n_{i}}^{(j)}\right. & =0, \omega)=\left[\begin{array}{l}
\underline{E_{\mathrm{Pf}}}\left(r_{n_{i}}^{(j)}=0, \omega\right) \\
\underline{E_{\mathrm{Pb}}}\left(r_{n_{i}}^{(j)}=0, \omega\right)
\end{array}\right] \\
& =M_{n_{i}}^{(j)} E_{\mathrm{Pout}}=\left[\begin{array}{ll}
M_{\mathrm{n}_{\mathrm{i} 11}}^{(j)} & M_{n_{i 12}}^{(j)} \\
M_{n_{i 21}}^{(j)} & M_{n_{i 22}}^{(j)}
\end{array}\right]\left(\frac{E_{T}}{0}\right),
\end{aligned}
$$

with

$$
\begin{gathered}
M_{n_{1}}^{(j)}=\left(\prod_{k=j}^{N} P_{n_{1}}^{(k)} S_{L} R_{12} P_{n_{2}}^{(k)} S_{L} R_{21}\right) R_{21}^{-1} R_{2 \mathrm{~S}}, \\
M_{n_{2}}^{(j)}=P_{n_{2}}^{(j)} S_{L} R_{21}\left[\prod_{k=j+1}^{N} P_{n_{1}}^{(k)} S_{L} R_{12} P_{n_{2}}^{(k)} S_{L} R_{21}\right] R_{21}^{-1} R_{2 S} \\
(\text { for } j \neq N), \\
M_{n_{2}}^{(N)}=P_{n_{2}}^{(N)} S_{L} R_{2 S}
\end{gathered}
$$

with the matrice for the whole structure given by

$$
M=R_{01}\left[\prod_{j=1}^{N} P_{n_{1}}^{(j)} S_{L} R_{12} P_{n_{2}}^{(j)} S_{L} R_{21}\right] R_{21}^{-1} R_{2 S} .
$$

In all these relations $R_{i j}$ is the interface transfer matrix between two media of indices $n_{i}$ and $n_{j}{ }^{19,20}$ (index 0 refers to incident media and index $S$ to the substrate media), and $P_{n_{i}}^{(j)}$ is the propagation matrix in the media of index $n_{i}$, and thickness $e_{i}^{(j)}=\lambda_{\mathrm{PC}} /\left(4 n_{i}\right)$ for quarter-wave layers (with $\lambda_{\mathrm{PC}}$ the central wavelength of the photonic band gap). ${ }^{19,20}$ We have extended this matrix to the case of absorbing media with intensity absorption $\alpha_{I}$, which gives 


$$
P_{n_{i}}^{(j)}=\left(\begin{array}{cc}
\exp \left\{-i\left[\left(2 \pi n_{i}\right) / \lambda\right] e_{i}^{(j)}\right\} \exp \left\{\left[\alpha_{i} e_{i}^{(j)}\right] / 2\right\} & 0 \\
0 & \exp \left\{i\left[\left(2 \pi n_{i}\right) / \lambda\right] e_{i}^{(j)}\right\} \exp -\left\{\left[\alpha_{i} e_{i}^{(j)}\right] / 2\right\}
\end{array}\right)
$$

The modeling also takes into account the scattering losses of the structure introduced through the matrix $S_{L}$ that models the influence of the quality and roughness of the interfaces. The scattering loss due to this roughness despite being small can have a strong influence owing to the large number of interfaces and light localization. This matrix is expressed as

$$
S_{L}=\left[\begin{array}{cc}
(1-a)^{-1} & 0 \\
0 & 1-a
\end{array}\right]
$$

where $a$ is the scattering-loss coefficient of each interface (supposed here to be the same for all the interfaces).

The $M$ matrix allows us to calculate the transmitted $E_{T}$ and reflected $E_{R}$ amplitudes for a given incident intensity $E_{I}$ (no signal is injected on the substrate side), whereas the $M_{n_{i}}^{(j)}$ gives the pump-beam amplitudes (used hereafter in the calculation of the nonlinear properties of the structure) and the localization factors in each layer for the forward and backward waves:

$$
\begin{gathered}
f_{\mathrm{Lf}}\left(n_{i}, j\right)=\frac{\underline{E_{P f}\left(r_{n_{i}}^{(j)}=0, \omega\right)}}{\frac{E_{I}}{-}}=\frac{M_{n_{i} 11}^{(j)}}{M_{11}}, \\
f_{\mathrm{Lb}}\left(n_{i}, j\right)=\frac{\underline{E_{P b}}\left(r_{n_{i}}^{(j)}=0, \omega\right)}{\underline{E_{I}}}=\frac{M_{n_{i} 21}^{(j)}}{M_{11}} .
\end{gathered}
$$

The measure of the light localization capability of the photonic crystal is defined by the average localization factor $f_{M}$, which gives the local-field enhancement of the forward-pump beam (with similar expressions for other considered beams) averaged on the whole structure, and that is expressed as:

$$
f_{M}=\frac{1}{2 N} \sum_{j=1}^{N}\left[f_{\mathrm{Lf}}\left(n_{1}, j\right) \sqrt{n_{1}}+f_{\mathrm{Lf}}\left(n_{2}, j\right) \sqrt{n_{2}}\right]
$$

Used with a bulk media (of index $n_{1}$ or $n_{2}$ ), this expression gives a reference value of 1 for $f_{M}$. As shown hereafter this localization factor is larger than unity at the band edge of photonic crystals and thus allows for considerable enhancement of the efficiency of nonlinear optical processes, such as the four-wave-mixing process studied in our analysis.

\section{Nonlinear Propagation Matrix of Signal} and Conjugate Beams

As in the linear case the left signal-field vector $E_{\operatorname{Sin}}$ is related to the right vector $E_{\text {Sout }}$ through a matricial relation using the nonlinear transmission matrix $M_{\mathrm{NL}}$ :

$$
\begin{aligned}
& \underline{E_{\mathrm{Sin}}}=\left(\begin{array}{c}
\underline{E_{\mathrm{Si}}} \\
\underline{E_{\mathrm{Sr}}} \\
\hline \underline{E_{\mathrm{Ci}}^{*}} \\
\hline \underline{E_{\mathrm{Cr}}^{*}}
\end{array}\right)=M_{\mathrm{NL}} \underline{E_{\mathrm{Sout}}}=\left(\begin{array}{cccc}
M_{11}^{\mathrm{NL}} & M_{12}^{\mathrm{NL}} & M_{13}^{\mathrm{NL}} & M_{14}^{\mathrm{NL}} \\
M_{21}^{\mathrm{NL}} & M_{22}^{\mathrm{NL}} & M_{23}^{\mathrm{NL}} & M_{24}^{\mathrm{NL}} \\
M_{31}^{\mathrm{NL}} & M_{32}^{\mathrm{NL}} & M_{33}^{\mathrm{NL}} & M_{34}^{\mathrm{NL}} \\
M_{41}^{\mathrm{NL}} & M_{42}^{\mathrm{NL}} & M_{43}^{\mathrm{NL}} & M_{44}^{\mathrm{NL}}
\end{array}\right) \\
& \times\left(\begin{array}{c}
\frac{E_{\mathrm{St}}}{0} \\
\frac{E_{\mathrm{Ct}}^{*}}{0}
\end{array}\right)
\end{aligned}
$$

that provides for a given input signal amplitude $E_{\mathrm{Si}}$, the reflected signal amplitude $E_{\mathrm{Sr}}$, the transmitted signal amplitude $E_{\mathrm{St}}$, the reflected conjugated amplitude $E_{\mathrm{Cr}}^{*}$, and the transmitted conjugated amplitude $E_{\mathrm{Ct}}^{*}$, supposing that no field is injected in the back side of the sample (Fig. 2) as well as in the conjugated field direction (meaning $E_{\mathrm{Ci}}^{*}$ $=0$ ). Using this matrix, the amplitude transmittivity $\frac{T_{S}}{T_{S}}$ and reflectivity $R_{S}$ of the signal beam, as well as the amplitude transmittivity $T_{C}^{*}$ and reflectivity $R_{C}^{*}$ of the conjugate beam, is expressed as

$$
\begin{aligned}
& T_{S}=\frac{E_{\mathrm{St}}}{\underline{\underline{E_{\mathrm{Si}}}}}=\frac{M_{33}^{\mathrm{NL}}}{M_{11}^{\mathrm{NL}} M_{33}^{\mathrm{NL}}-M_{13}^{\mathrm{NL}} M_{31}^{\mathrm{NL}}}, \\
& R_{S}=\frac{E_{\mathrm{Sr}}}{\underline{\underline{E_{\mathrm{Si}}}}}=T_{S}\left[\frac{M_{21}^{\mathrm{NL}} M_{33}^{\mathrm{NL}}-M_{23}^{\mathrm{NL}} M_{31}^{\mathrm{NL}}}{M_{33}^{\mathrm{NL}}}\right], \\
& T_{C}^{*}=\frac{E_{\mathrm{Ct}}^{*}}{\overline{E_{\mathrm{Si}}}}=-T_{S} \frac{M_{31}^{\mathrm{NL}}}{M_{33}^{\mathrm{NL}}}, \\
& R_{C}^{*}=\frac{E_{\mathrm{Cr}}^{*}}{\overline{E_{\mathrm{Si}}}}=T_{S}\left[\frac{M_{41}^{\mathrm{NL}} M_{33}^{\mathrm{NL}}-M_{43}^{\mathrm{NL}} M_{31}^{\mathrm{NL}}}{M_{33}^{\mathrm{NL}}}\right] .
\end{aligned}
$$

As previously for the pump beam, the nonlinear transmission matrix $M_{\mathrm{NL}}$ for the $1 \mathrm{D}$ photonic crystal is expressed as

$$
M_{\mathrm{NL}}=R_{01}^{S}\left[\prod_{j=1}^{N} P_{n_{1}}^{\mathrm{NL}(j)} S_{\mathrm{NL}} R_{12}^{S} P_{n_{2}}^{\mathrm{NL}(j)} S_{\mathrm{NL}} R_{21}^{\mathrm{S}}\right] R_{21}^{S^{-1}} R_{2 \mathrm{~S}}^{S}
$$

with the nonlinear propagation matrices $P_{n_{i}}^{\mathrm{NL}}(j)$, the reflection matrices $R_{i j}^{S}$, and the scattering loss matrix $S_{\mathrm{NL}}$ given in Appendix A.

Using a process similar to the one used for the pumpbeam propagation, we are also able to easily extract from the matricial model the amplitude of the signal and conjugate beam in each layer and see their repartition 
throughout the photonic crystal, which is very important for verifying the validity of the SVEA approximation made in our analysis.

\section{FOUR-WAVE MIXING IN A PERFECT 1D PHOTONIC CRYSTAL}

Using the model described in Subsection 2.B we can simulate the characteristics of degenerate four-wave mixing in a 1D photonic crystal. As a basis of calculation we take a stack made of two $\lambda / 4$ optically thick materials of index $n_{1}=3$ and $n_{2}=2.5$, corresponding approximately to the index contrast of III-V or II-VI semiconductor structures. For the sake of simplicity, the refractive index (and nonlinear susceptibility) dispersion is neglected here. Indeed, its inclusion, possible within the model, would not drastically change the main conclusions that we can deduce from the simulations. As an example, the wavelength range of operation of the device considered in our analysis is in the 700-900 $\mathrm{nm}$, with a bandgap center wavelength located at $\lambda_{\mathrm{PC}}=800 \mathrm{~nm}$. To simplify the analysis and get results that are easily interpreted, in this first step we consider a perfect photonic crystal without any losses, i.e., neglecting scattering losses at the interface $(a=0)$ and absorption losses in the layers $\left(\alpha_{1}=\alpha_{2}=0\right)$.

\section{A. Light Localization in a 1D Photonic Crystal}

We first consider a simple 1D photonic crystal and calculate with the model the repartition of the optical field in-

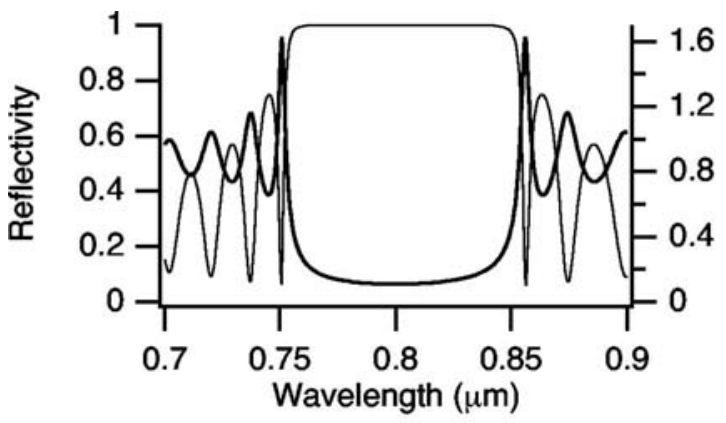

(a)

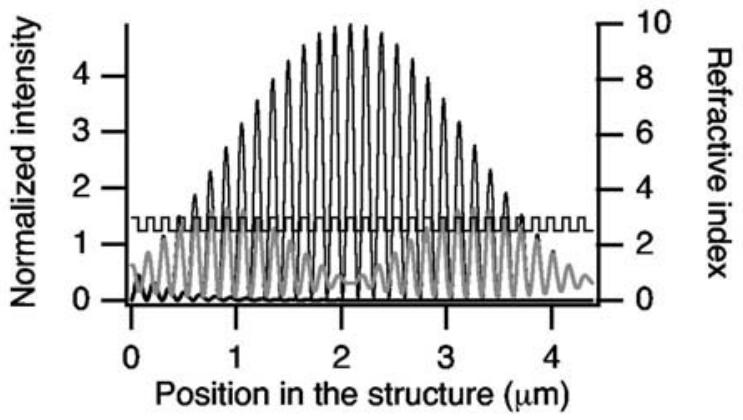

(b)

Fig. 3. (a) Reflectivity and average localization factor (thick curve) of a 1D photonic crystal of 30 pairs of layers. (b) Repartition of light intensity normalized to incident intensity inside the structure at different positions in the spectra; in the center of the band gap (thick curve), at the first minima of the band edge (thin curve) and at the second minima of the band edge (gray curve); in addition, the index structure is presented.

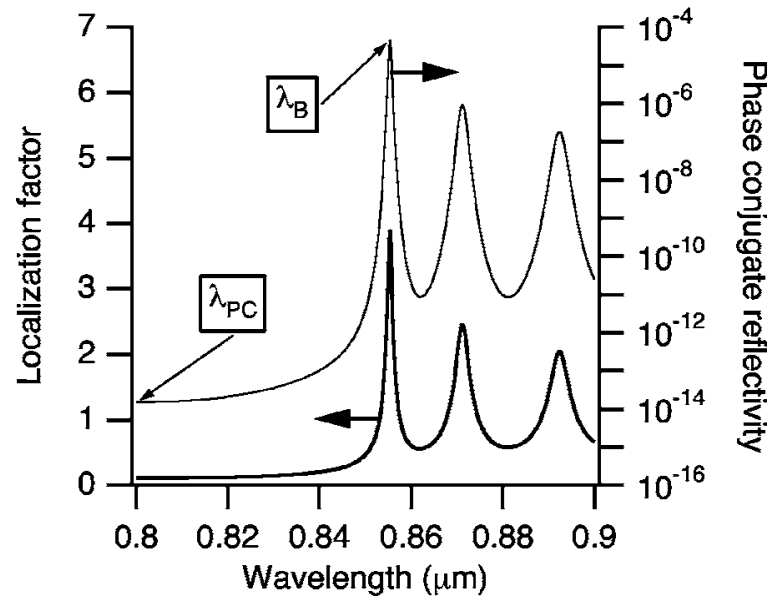

Fig. 4. Phase-conjugate reflectivity (thin curve) and average localization factor (thick curve) of a 1D photonic crystal of 30 pairs of layers centered at $\lambda_{\mathrm{PC}}$ beyond a 20 pair Bragg mirror centered at $\lambda_{\mathrm{B}}$. The pump intensity in these calculations is set to $10 \mathrm{MW} \mathrm{cm}{ }^{-2}$.

side the structure. The structure is composed of 30 pairs of layers. The reflectivity of such a structure shows a typical forbidden bandgap in which reflectivity reaches 1 , and oscillations of the reflectivity ${ }^{18,20}$ on the edge of the bandgap due to the finite size of the structure [Fig. 3(a)]. At the center of the bandgap $(\lambda=800 \mathrm{~nm})$, the light penetrates only the first layers of the structure [see thick curve in Fig. 3(b)], and intensity decreases very rapidly inside the photonic crystal. At the band edge, the structure again becomes transparent with a low reflectivity, which would be zero for left and right surrounding media made of the same materials as the structure. This high transmission of the structure (at $\lambda=856.4 \mathrm{~nm}$ ) coincides with a peak value of the average localization factor $f_{M}$ [see the thick curve line in Fig. 3(a)] as the transmitted light goes out of the structure only after having stayed a long time owing to multiple reflections on the layer interfaces. The intensity repartition shown in the thin curve of Fig. 3(b) demonstrates that locally the intensity in the structure (normalized to the input intensity) can be much higher than that injected by a factor that can reach 5 . This light localization also exists on the short-wavelength edge of the bandgap, but there the maxima of the stationary wave existing in the structure would be localized in the low-index region instead of the high-index region. ${ }^{1}$ Light localization exists also at other minima of the reflectivity [see gray curve in Fig. 3(b)] but then with lower localization and with multipeak repartition (as can be seen for the second minima at $\lambda=874.4 \mathrm{~nm}$ ).

B. Phase-Conjugate Reflectivity of a 1D Photonic Crystal As shown in Subsection 2.A, the phenomenon of light localization is very favorable to the nonlinear efficiency of the structure. However, using a simple photonic crystal, localization occurs when the photonic crystal becomes transparent, which means that the forward-pump beam of four-wave mixing is not generated efficiently (even if it yet exists in each layer, owing to multiple reflections). It is thus very favorable to have a mirror at the exit of the photonic crystal that reflects the transmitted beam to ef- 


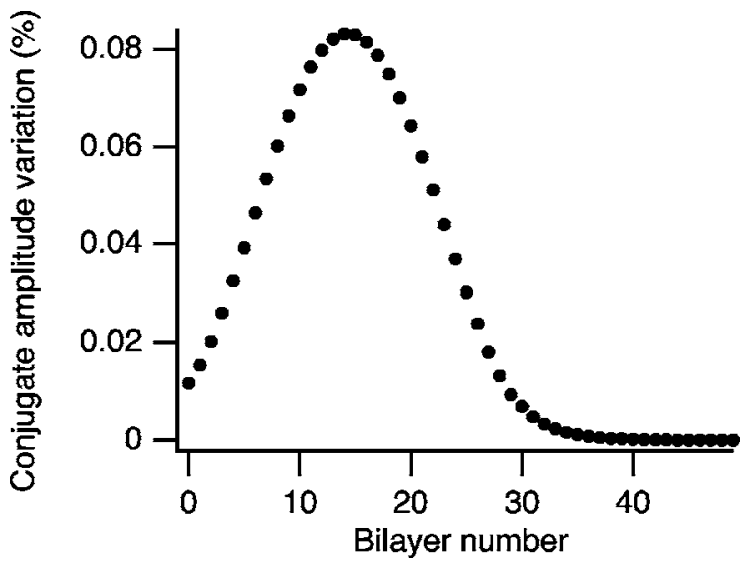

Fig. 5. Evolution of the variation of the amplitude of the forward-phase conjugate wave in the low index layers of the photonic crystal for the phase-conjugate-reflectivity peak wavelength. The pump intensity in these calculations is set to $10 \mathrm{MW} \mathrm{cm}^{-2}$.

ficiently generate the backward-pump beam. This mirror is also necessary to generate the backward-pump beam in the calculation for the bulk structure, which allows a quantitative measurement of the nonlinearity enhancement brought by the structuring of the material. The easiest way to implement this mirror, both for simulation and fabrication of the structure, is to place at the exit of the photonic-crystal structure a Bragg mirror with its center wavelength positioned at the band-edge wavelength of the photonic crystal (this is simply done by changing slightly the thickness of the layers). In the present structure the Bragg mirror is then centered at $\lambda_{B}=856.4 \mathrm{~nm}$ (increasing the thicknesses of the layers by a ratio $\lambda_{B} / \lambda_{\mathrm{PC}}$ ), and has 20 pairs of layers, composed of the same materials as the studied photonic crystal.

For the calculation, the two media of the photonic crystal have identical nonlinear coefficients that are equal to $D \chi_{0_{i}}^{(3)}=-1.7 \times 10^{-11}$ esu (which corresponds to the order of magnitude of the value encountered in II-VI and III-V semiconductor compounds ${ }^{16}$ ).

Spectra of Fig. 4 clearly demonstrate that the peak of the localization factor positioned at the edge of the bandgap (thick curve) corresponds to a peak of the phaseconjugate reflectivity, with the secondary peaks already observed for all the rebounds of the band-edge reflectivity [Fig. 3(a)]. Light localization is increased compared with the situation in the absence of the Bragg mirror, as light transmitted by the photonic crystal is reflected almost completely by the second Bragg mirror and participates with the four-wave-mixing mechanism. For this calculation $f_{M}$ given by Eq. (11) is calculated only in the photonic crystal, without taking into account the Bragg mirror in which light does not penetrate in the considered wavelength range. The gain in phase-conjugate reflectivity brought by the structuring reaches almost 4 orders of magnitude compared with the equivalent thickness of bulk media, which immediately shows the interest of the structuring of the material, for nonlinear functions.

\section{Validity of the SVEA}

All the calculations presented here were made with a model using nonlinear propagation equations established under the SVEA, which stipulates low relative modifications of the field amplitudes for a propagation length of $\lambda$. This problem is therefore of importance for photonic crystals having modulation periods smaller than $\lambda$. In fact, it has already been shown that this approximation could be invalid in the special case of the Kerr effect in 1D photonic crystals, ${ }^{15}$ at a high pump power when bistability phenomenon is predicted. To verify that the approximation is valid in our device modeled well below the bistability threshold, the phase-conjugate amplitudes are calculated at the input and the output of each nonlinear layer of the structure. Figure 5 shows the evolution of the relative variation of the conjugate amplitude in each layer (in fact, the difference of these amplitudes normalized to their sum) inside the structure. The case of the forward phaseconjugate wave in the low-index media is shown in Fig. 5, as it gives the highest variation (the low-index media is thicker, in the $\lambda / 4$ structure), and because all the other curves have the same shape. The spatial repartition shown in Fig. 5 shows that the maximum variation is smaller than $0.1 \%$ on a thickness of $\lambda / 4$, which means that the SVEA is actually verified for the four-wavemixing phenomena in a 1D photonic crystal in the regime where bistability phenomena can be neglected.

\section{Influence of Structure Parameters}

In this section the evolution of the phase-conjugate reflectivity of the device is considered as a function of structure parameters (number of layers and index contrast) or experimental conditions (incidence angle of the signal beam). The influence of the number of layers of the photonic crystal (for the same number of layers of the back Bragg mirror, i.e., 20 pairs of layers) is shown in Fig. 6 showing the peak phase-conjugate reflectivity as a func-

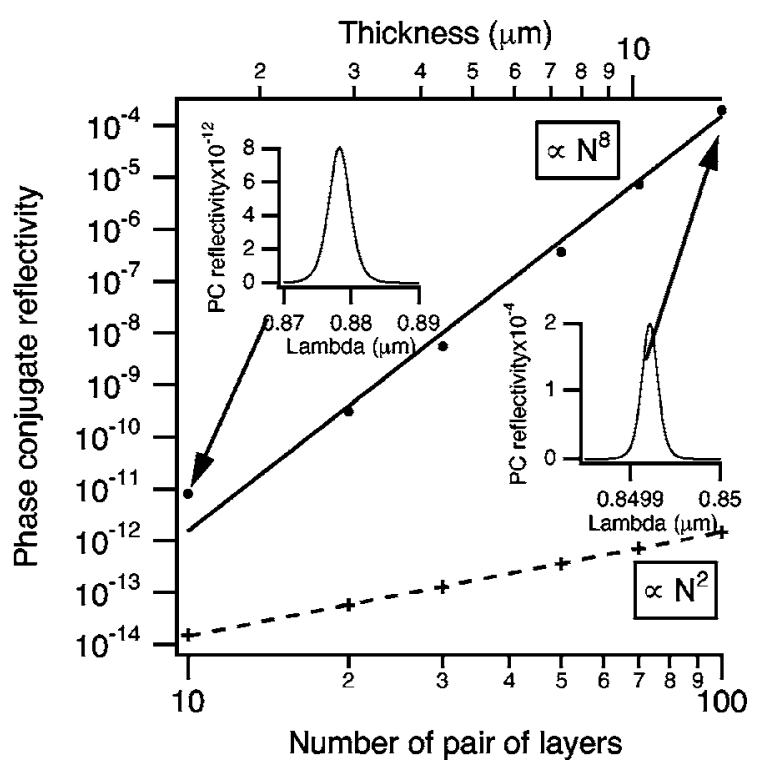

Fig. 6. Phase-conjugate reflectivity of the photonic crystal (filled circles) and of an equivalent-thickness bulk crystal (crosses) as a function of the number of pairs of layers $N$ (or the thickness of the structure). Curves represent the theoretical variation with the number of pairs of layers. The insets show the phaseconjugate-reflectivity spectra of the photonic crystal for some peculiar points. The pump intensity in these calculations is set to $0.1 \mathrm{MW} \mathrm{cm}^{-2}$. 


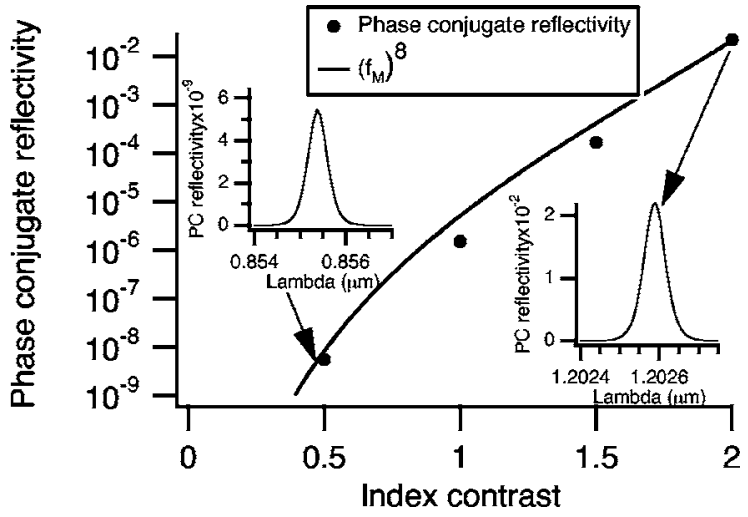

Fig. 7. Phase-conjugate reflectivity of the photonic crystal (filled circles) as a function of the index contrast (with $n_{1}=3$ ). The curve represents the theoretical variation with the index contrast given by Eq. (16). The insets show the phase-conjugate-reflectivity spectra for some peculiar points. The pump intensity in these calculations is set to $0.1 \mathrm{MW} \mathrm{cm}^{-2}$.

tion of the number of layer pairs. A very strong increase of the phase-conjugate reflectivity with the eighth power of the number of layers pairs is shown in Fig. 6. For the bulk media (deposited on the same Bragg mirror to generate the forward-pump beam), in the same condition, the growth is only to the second power of the crystal thickness. ${ }^{14,17}$ The small departure to the eighth-power variation at small thickness (or low number of pairs of layers), is due to the influence of the Bragg mirror that is also made of nonlinear materials. Light just penetrates the first layers of this Bragg mirror, and the relative contribution of these layers to phase-conjugate reflectivity is significant for small numbers of bilayers but decreases rapidly as the number of layers of the photonic crystal increases.

This variation with the eighth power of $N$ layer pairs can be easily understood, reminding us that the effective nonlinear coefficient varies as the fourth power of the localization factor [as shown by Eq. (4b) for identical beams], which gives the eighth power for the phaseconjugate reflectivity. This localization factor is proportional to the number of layer pairs that can be calculated analytically from the group-velocity expression in a photonic crystal around the band edge ${ }^{18}$ :

$$
\begin{aligned}
f_{M} & =\left[\frac{1-T_{12} \cos ^{2}(\pi / 2 N)}{T_{12} \sin ^{2}(\pi / 2 N)}\right]^{1 / 2} \underset{\mathrm{N} \rightarrow+\infty}{\rightarrow} \frac{2}{\pi}\left(\frac{1-T_{12}}{T_{12}}\right)^{1 / 2} N \\
& =\frac{\Delta n}{\sqrt{n_{1} n_{2}}} \frac{N}{\pi}
\end{aligned}
$$

where $T_{12}=4 n_{1} n_{2} /\left(n_{1}+n_{2}\right)^{2}$ is the intensity-transmission factor between the media of index $n_{1}$ and $n_{2}$ and $\Delta n=n_{1}$ $-n_{2}$ is the index contrast.

It must be also underlined that the large increase in the optical nonlinearity is accompanied by a narrowing of the phase-conjugate reflectivity spectrum, as shown in the insets of Fig. 6 with a reduction of the phaseconjugate reflectivity linewidth by a factor of 1700 . This result is in accordance with the general concept of resonances, as in the case of Fabry-Perot cavities.
Since the localization factor also is dependant on the index contrast between the two layers [see Eq.(16)], so is the phase-conjugate reflectivity. This variation is shown in Fig. 7 for the previous structure (with a 30 layer-pairs photonic crystal, and a 20 layer-pairs Bragg mirror), where the low-index value is varied down to 1 (and the high index keeps its value $n_{1}=3$ ). For each value of the index contrast, the position of the Bragg mirror is adjusted to compensate for the variation of the position of the band edge, with the index contrast. The phaseconjugate reflectivity (solid circles) increases with the index contrast by several orders of magnitude. This variation is compared with the theoretical expression of the localization factor given by Eq. (16), by tracing the evolution of the eighth power of this parameter, which shows a good accordance between the calculations. We can note here that, as in the case of the variation with the number of layers, this increase is accompanied by a drastic reduction of the width of the peaks (see insets in Fig. 7) that may become smaller than the pulse spectrum if shortduration pulses are used, giving some limits in the experimental use of such an effect with too-short-duration pulses.

All the results presented here suppose that all the beams were incident normally to the structure. Experimentally, the necessity to extract the phase-conjugate beam may impose an angle between the signal and pump beams. The calculation predicts the tolerance on this small angle. Figure 8 shows the variation of the phaseconjugate reflectivity as a function of the incidence angle $\theta$ of the signal beam. A strong decrease by nearly four orders of magnitude is demonstrated when $\theta$ increases to $20^{\circ}$, followed by a second peak located at $32^{\circ}$ with a drop in efficiency by only 1 order of magnitude. Indeed, when the incidence angle of the signal beam increases, an increase of the optical thickness of the material arises, giving a blueshift of the reflectivity curve of the signal beam compared with that of the pump beam. The band edge for the signal beam is then shifted toward a smaller wavelength and is no longer superimposed with that of the

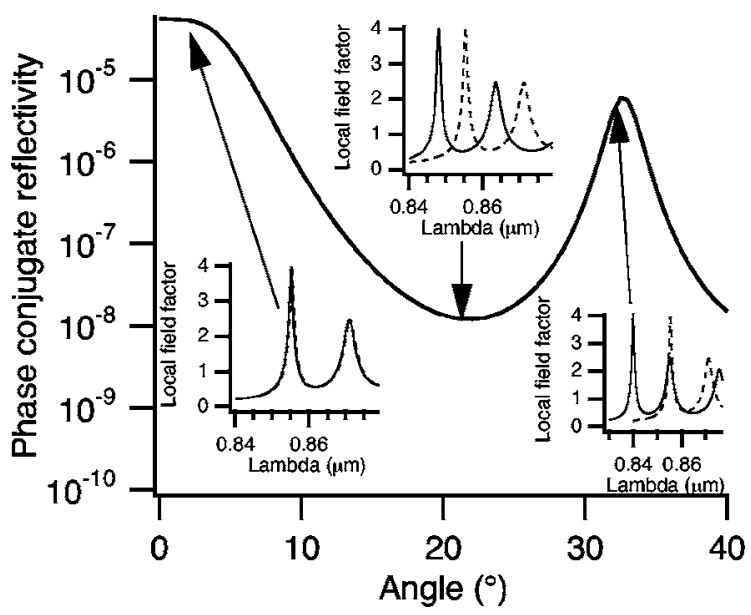

Fig. 8. Phase-conjugate reflectivity of the photonic crystal as a function of the incident angle of the signal beam. The insets show the local field factor spectra of the pump beam (dashed curve) and the signal beam (solid curve) for some peculiar angles. The pump intensity in these calculations is set to $10 \mathrm{MW} \mathrm{cm}^{-2}$. 


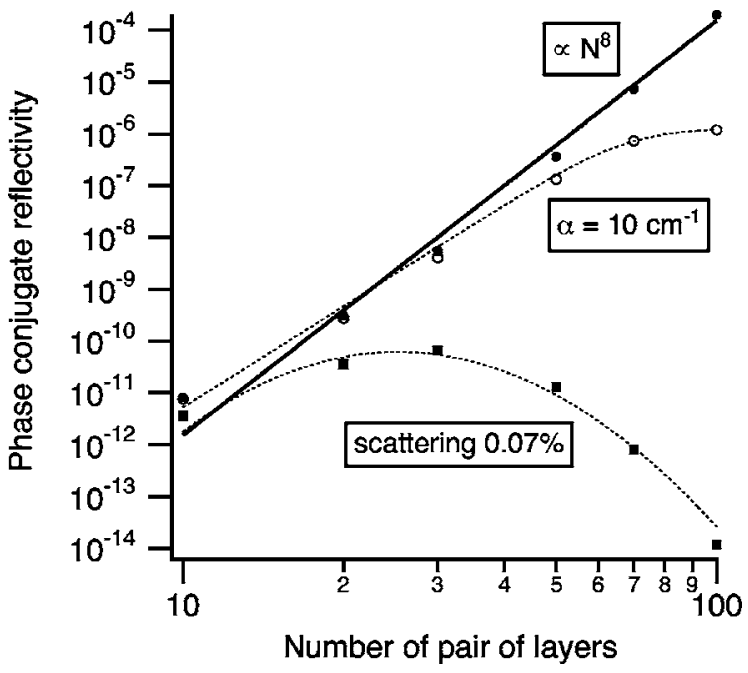

Fig. 9. Influence of losses on the phase-conjugate reflectivity of the photonic crystal as a function of the number of pairs of layers $N$ for scattering losses (filled squares) of $0.07 \%$ per interface and for absorption losses (empty circles) of $10 \mathrm{~cm}^{-1}$ for each media of the structure, with the reference curve without losses (filled circles). For both curves with losses, the dotted curves are guidelines for the eyes. The pump intensity in these calculations is set to $0.1 \mathrm{MW} \mathrm{cm}{ }^{-2}$.

pump beam. The phase-conjugate efficiency decreases rapidly to a very small value, as light localized for the pump beam at a used wavelength is localized for a different wavelength for the signal beam (see insets in Fig. 8), leading to different values of $f_{i}$ for the different beams in Eq. (4b). Nevertheless, the phase-conjugate signal reappears at large angles when the second band-edge peak of the signal beam coincides with the first peak of the pump beam, but as light localization is less efficient for this second peak [see Fig. 3(b)], this second phase-conjugate reflectivity peak is $\sim 1$ order of magnitude smaller than the peak obtained at a small angle.

We also deduce from this curve that at a low angle the phase-conjugate reflectivity varies rather smoothly, as it decreases only by a factor of two for an angle of $4.5^{\circ}$, and no significant decrease of the signal is seen for an incident angle of the signal beam of $2^{\circ}$, which is important from an experimental point of view, since a small angle between the beams can be tolerated, allowing an easy extraction of the phase-conjugate beam. ${ }^{21}$

\section{INFLUENCE OF LOSSES ON THE PHASE-CONJUGATE REFLECTIVITY OF A 1D PHOTONIC CRYSTAL}

All the curves until now have been calculated supposing that the structures were perfect, i.e., lossless. The presence of losses may modify the obtained results, as light localization also corresponds to an increase of the influence of the losses due to the multiple reflexions on each internal interface of the structure. As the photons trapped in the structure travel a long time inside it, they see several times the losses before leaving it, exacerbating their influence.

In the evaluated photonic crystals, losses may have two origins: scattering at the interface between two different layers and absorption losses inside the layers themselves. For the calculation, we separate both cases as they differ in their influence on the phase-conjugate reflectivity. The first cause for losses is the absorption of the media that compose the photonic crystal. We take for the calculation a constant absorption of $10 \mathrm{~cm}^{-1}$ for both media. This value is rather large and would correspond to a bulk media operated rather close to its electronic bandgap or to a material with a two-photon absorption coefficient of $40 \mathrm{~cm} \mathrm{GW}^{-1}$ operated with an intensity $250 \mathrm{MW} \mathrm{cm}{ }^{-2}$ (corresponding, for example, to pulses of high fluence of $2.5 \mathrm{~mJ} \mathrm{~cm}^{-2}$ with a $10 \mathrm{ps}$ duration). The second cause of losses is linked to the quality of the interfaces (especially their roughness) existing in the 1D photonic-crystal structures. This roughness causes very low scattering of light, but has a strong influence owing to the large number of interfaces and to light localization. Regarding the highquality state-of-the-art structures based on II-VI materials such as $\mathrm{CdMnTe}-\mathrm{CdMgTe}$ microcavities, residual ruggedness induces a loss of the order of $0.07 \%$ per interface. $^{22}$

Figure 9 compares the phase-conjugate reflectivities plotted as a function of the number of layer pair for no losses (solid circles), absorption losses (open circles) and scattering losses (square). In the case of absorption losses, the reflectivity continues to increase for large number of layers, with a saturation that begins to appear around 100 pairs of layers. This means that the phase-conjugate reflectivity is rather tolerant to the presence of absorption in the media. On the other hand, the influence of scattering losses is very strong, since the phase-conjugate reflectivity increases until about $N=30$ pairs of layers then decreases, until it is smaller than the reflectivity of a bulk material of the same thickness for $N>70$. This means that for such a level of scattering losses the best compromise is around 30 pairs of layers, with a phase-conjugate reflectivity that will be increased by 2 orders of magnitude compared with bulk media (instead of 4 orders of magnitude for a perfect structure).

We also calculated the influence of scattering losses on the angular variation (see Fig. 10). As expected from Fig.

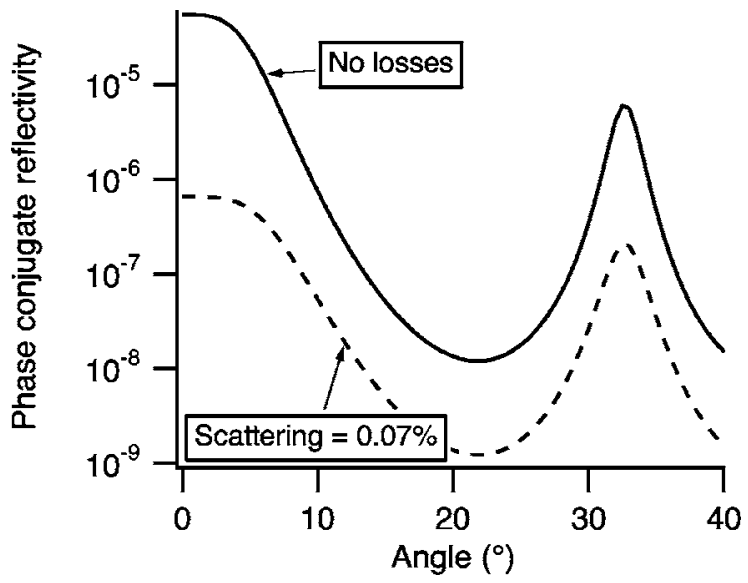

Fig. 10. Phase-conjugate reflectivity of the photonic crystal (solid curve) as a function of the incident angle of the signal beam. The dashed curve represents the same calculation taking into account scattering losses $(0.07 \%$ per interface). The pump intensity in these calculations is set to $10 \mathrm{MW} \mathrm{cm}^{-2}$. 
9 a strong decrease of 2 orders of magnitude appears for the zero-angle peak, but a smaller decrease (around 1 order of magnitude) is observed for the large-angle peak. This observation is easily explained, as for this peak the signal-beam localization is less efficient and thus less sensitive to losses, giving a smaller reduction of the phaseconjugate reflectivity.

In any case the results presented in this section definitely prove that losses are main determinants of the efficiency of nonlinear processes in photonic crystals. The case of scattering losses is particularly important, demonstrating the need of very good interfaces between layers having the maximum possible index contrast.

\section{CONCLUSION}

We have presented in this paper a model for the description of degenerate four-wave-mixing phenomena in a $1 D$ photonic crystal. This model is based on a nonlinear extension of the transfer-matrix description of beam propagation in the structure. It has been used to model the influence of light localization on the enhancement of the phase-conjugate reflectivity near the band edge of the structure. An increase of reflectivity varying as the eighth power of the number of layers is expected, as well as a large dependence on the index contrast of the structure. Both of those phenomenon are accompanied by a strong reduction of the resonance width, which may lead to some limitation of the enhancement when ultrashort-duration pulses are used. The model also shows a strong influence of the losses on the nonlinear efficiency of the structure, an influence that is shown to be greater for scattering losses than for absorption losses for usual structures. The transfer-matrix description is also shown to be compatible with the SVEA, despite the small dimension of the structures constituting the photonic crystal. Those results, despite being obtained for a 1D photonic crystal, give hints of the optimization of more-complex structures like twodimensional photonic crystals, for which modeling of nonlinear phenomena is still missing.

The developed model is also very versatile and can be applied very easily to complex structures as each layer is defined by its own parameters (thickness, index, and nonlinear coefficient). The model then allows us to insert defects in the structure, form microcavities or coupled microcavities, or model stacks of photonic crystals with different parameters or even model in extreme cases nonperiodic structures with media having various refractive indices and thicknesses.

Further improvement of the model will go in the direction of the inclusion of other nonlinear effects, such as Kerr nonlinearity or two-photon absorption, in order to go beyond the low-efficiency regime modeled here. Another direction is the modeling of nondegenerate four-wave mixing and its application for wavelength conversion, as well as the extension of the transfer-matrix modeling towards the description of two-dimensional photonic-crystal structures.

\section{APPENDIX A}

In each layer of the structure a four-wave-mixing phenomenon occurs that couples the forward signal beam to the backward conjugate beam (propagating with an angle $+\theta$ ) and the backward signal beam to the forward conjugate beam (propagating with an angle $-\theta$ ), through interaction with the forward and backward pump beams (see Fig. 2). For the modeling we define the complex amplitude of the fields as $E_{I j}(r, \omega)=\hat{e}_{I j} A_{I j}(r, \omega) \exp i k_{I j} r$ with $\hat{e}_{I j}$ as the polarization vector of the wave $I j(I=P, S$ and $C$, for the pump, the signal and the conjugate beams, and $j=f$ and $b$ for the forward and backward propagating waves); $A_{I j}(r, \omega)$ as its amplitude; and $k_{I j}$ as its wave vector of direction $\hat{k}_{I j}$. In the SVEA, ${ }^{14,17}$ we obtain two systems of coupled equations (in cgs units):

$$
\begin{aligned}
\hat{k}_{\mathrm{Sf}} \cdot \nabla A_{\mathrm{Sf}}(r, \omega)= & i \frac{4 \pi^{2}}{n \lambda} D\left[\hat{e}_{C} \cdot \underline{\underline{\bar{\chi}}}(\omega, \omega,-\omega) \hat{e}_{F} \hat{e}_{B} \hat{e}_{S}\right] A_{\mathrm{Pf}}(r, \omega) \\
& \times A_{\mathrm{Pb}}(r, \omega) A_{\mathrm{Cb}}^{*}(r, \omega)-\frac{\alpha}{2} A_{\mathrm{Sf}}(r, \omega), \\
\hat{k}_{\mathrm{Cb}} \cdot \underline{\nabla} A_{\mathrm{Cb}}(r, \omega)= & i \frac{4 \pi^{2}}{n \lambda} D\left[\hat{e}_{C} \cdot \underline{\underline{\underline{\chi}}}(\omega, \omega,-\omega) \hat{e}_{F} \hat{e}_{B} \hat{e}_{S}\right] \\
& \times A_{\mathrm{Pf}}(r, \omega) A_{\mathrm{Pb}}(r, \omega) A_{\mathrm{Sf}}^{*}(r, \omega)-\frac{\alpha}{2} A_{\mathrm{Cb}}(r, \omega),
\end{aligned}
$$

for the forward-signal and backward-conjugate waves, and an identical system of equations for the backwardsignal and forward-conjugate wave, simply changing $A_{\mathrm{Sf}}$ in $A_{\mathrm{Sb}}$ (and $\hat{k}_{\mathrm{Sf}}$ in $\hat{k}_{\mathrm{Sb}}$ ) and $A_{\mathrm{Cb}}$ in $A_{\mathrm{Cf}}$ (and $\hat{k}_{\mathrm{Cb}}$ in $\hat{k}_{\mathrm{Cf}}$ ).

In these equations, $\hat{k}_{\mathrm{Cb}}=-\hat{k}_{\mathrm{Sf}}=(-\sin \theta, 0,-\cos \theta)$, whereas $\hat{k}_{\mathrm{Cf}}=-\hat{k}_{\mathrm{Sb}}=(-\sin \theta, 0, \cos \theta)$, according to the different orientation of the beams in the problem, and $\alpha$ is the intensity absorption coefficient of the media. Solving Eq. (A1) for plane waves propagating in an infinite medium in the direction perpendicular to the propation direction, ${ }^{17}$ one can relate the field vector at the entrance of each nonlinear medium (of index $n_{i}$ ) to the field vector at the output through the nonlinear propagation matrix $P_{n_{i}}^{\mathrm{NL}(j)}$ defined by

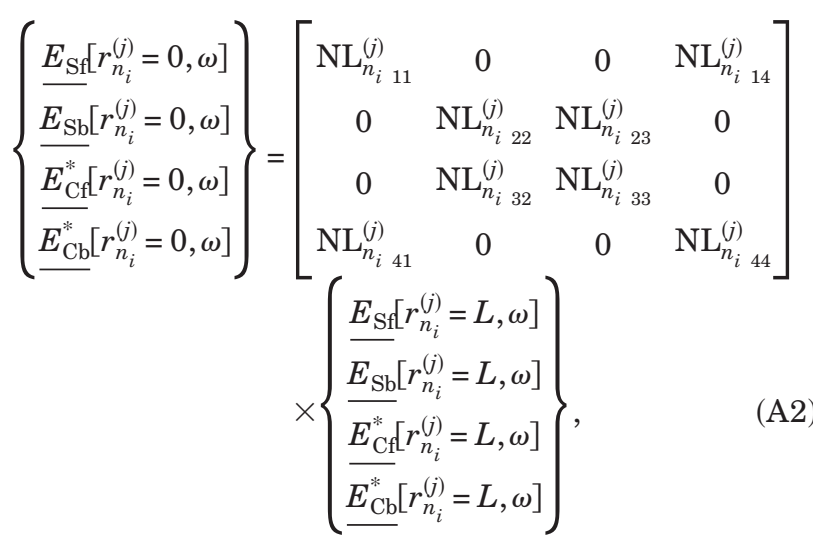




$$
\begin{aligned}
& \mathrm{NL}_{n_{i 11}}^{(j)}=\mathrm{NL}_{n_{i 33}}^{(j)^{*}}=\left\{\cos \left[\alpha_{n_{i}}^{\prime(j)} e_{i}^{(j)}\right]+\frac{\alpha_{\mathrm{i}} e_{i}^{(j)}}{2} \frac{\sin \left[\alpha_{n_{\mathrm{i}}}^{\prime \prime(j)} \mathrm{e}_{\mathrm{i}}^{(j)}\right]}{\alpha_{n_{i}}^{\prime(j)} e_{i}^{(j)}}\right\} \\
& \times \exp \left[-i \frac{2 \pi n_{i}}{\lambda} e_{i}^{(j)} \cos \theta_{i}\right] \text {, } \\
& \mathrm{NL}_{n_{i 44}}^{(j)}=\mathrm{NL}_{n_{i 22}}^{(j)^{*}}=\left\{\cos \left[\alpha_{n_{i}}^{\prime \prime(j)} e_{i}^{(j)}\right]-\frac{\alpha_{i} e_{i}^{(j)}}{2} \frac{\sin \left[\alpha_{n_{i}}^{\prime \prime(j)} e_{i}^{(j)}\right]}{\alpha_{n_{i}}^{\prime(j)} e_{i}^{(j)}}\right\} \\
& \times \exp \left[-i \frac{2 \pi n_{i}}{\lambda} e_{i}^{(j)} \cos \theta_{i}\right] \text {, } \\
& \mathrm{NL}_{n_{i 14}}^{(j)}=\mathrm{NL}_{n_{i}{ }_{32}}^{(j)^{*}}=-\mathrm{i}\left[\alpha_{n_{i}}^{\prime(j)} e_{i}^{(j)}\right] \exp \left[i \phi_{n_{i}}^{(j)}\right]\left\{\frac{\sin \left[\alpha_{n_{\mathrm{i}}}^{\prime \prime(j)} e_{i}^{(j)}\right]}{\alpha_{n_{i}}^{\prime \prime(j)} e_{i}^{(j)}}\right\} \\
& \times \exp \left[-i \frac{2 \pi n_{i}}{\lambda} e_{i}^{(j)} \cos \theta_{i}\right], \\
& \mathrm{NL}_{n_{i 41}}^{(j)}=\mathrm{NL}_{n_{i}{ }^{2}}^{(j)^{*}}=-\mathrm{i}\left[\alpha_{n_{i}}^{\prime(j)} e_{i}^{(j)}\right] \exp \left[-i \phi_{n_{i}}^{(j)}\right]\left\{\frac{\sin \left[\alpha_{n_{i}}^{\prime \prime(j)} e_{i}^{(j)}\right]}{\alpha_{n_{i}}^{\prime \prime(j)} e_{i}^{(j)}}\right\} \\
& \times \exp \left[-i \frac{2 \pi n_{i}}{\lambda} e_{i}^{(j)} \cos \theta_{i}\right],
\end{aligned}
$$

where $\alpha_{n_{i}}^{\prime \prime(j)}, \alpha_{n_{i}}^{(j)}$ and $\phi_{n_{i}}^{(j)}$ are defined in each layer of index $n_{i}$ and bilayer position $(j)$ by relations

$$
\begin{aligned}
& \alpha_{n_{i}}^{\prime \prime(j)^{2}}=\alpha_{n_{i}}^{\prime(j)^{2}}-\left(\frac{\alpha_{i}}{2}\right)^{2}, \\
& \alpha_{n_{i}}^{\prime(j)}=\frac{4 \pi^{2}}{n_{i} \lambda \cos \theta} D\left|\chi_{0_{i}}^{(3)}\right| \underline{E_{\mathrm{Pf}}}\left[r_{n_{i}}^{(j)}=0, \omega\right]\left|\underline{E_{\mathrm{Pb}}}\left[r_{n_{i}}^{(j)}=0, \omega\right]\right|, \\
& \exp \left[i \phi_{n_{i}}^{(j)}\right]=\frac{\chi_{0_{i}}^{(3)} \underline{E_{\mathrm{Pf}}}\left(r_{n_{i}}^{(j)}=0, \omega\right) \underline{E_{\mathrm{Pb}}}\left(r_{n_{i}}^{(j)}=0, \omega\right)}{\left|\chi_{0_{i}}^{(3)}\right| \underline{E_{\mathrm{Pf}}}\left(r_{n_{i}}^{(j)}=0, \omega\right)\left|\underline{E_{\mathrm{Pb}}}\left(r_{n_{i}}^{(j)}=0, \omega\right)\right|},
\end{aligned}
$$

using the pump beam amplitudes at the entrance of the layer calculated previously through Eqs. (10).

The signal beam reflection matrices $R_{i j}^{S}$ is defined as

$$
R_{i j}^{S}=\frac{1}{t_{n_{i} n_{j}}}\left(\begin{array}{cccc}
1 & r_{n_{i} n_{j}} & 0 & 0 \\
r_{n_{i} n_{j}} & 1 & 0 & 0 \\
0 & 0 & 1 & r_{n_{i} n_{j}} \\
0 & 0 & r_{n_{i} n_{j}} & 1
\end{array}\right),
$$

where

for the TE-polarized beams considered in our analysis, propagating with angle $\theta_{i}$ and $\theta_{j}$ in the media of index $n_{i}$ and $n_{j}$, respectively. These angles are related by the refraction relation $n_{i} \sin \theta_{i}=n_{j} \sin \theta_{j}=\sin \theta$ to the incident signal beam angle $\theta$.

The scattering loss matrix $S_{\mathrm{NL}}$ is expressed, similarly to $S_{L}$, as

$$
S_{\mathrm{NL}}=\left(\begin{array}{cccc}
(1-a)^{-1} & 0 & 0 & 0 \\
0 & 1-a & 0 & 0 \\
0 & 0 & (1-a)^{-1} & 0 \\
0 & 0 & 0 & 1-a
\end{array}\right)
$$

P. Delaye is the corresponding author and can be reached by telephone at 33169358750 , by fax at 33169 3587 00, or by email at philippe.delaye@iota.u-psud.fr.

\section{REFERENCES}

1. J. D. Joannopoulos, R. D. Meade, and J. N. Winn, Photonic Crystals (Princeton U. Press, 1995).

2. T. F. Krauss and R. M. De La Rue, "Photonic crystals in the optical regime-past, present and future," Prog. Quantum Electron. 23, 51-96 (1999).

3. W. Chen and D. L. Mills, "Optical response of nonlinear multilayer structures: bilayers and superlattices," Phys. Rev. B 36, 6269-6278 (1987).

4. Y. Dumeige, I. Sagnes, P. Monnier, P. Vidakovic, C. Meriadec, and A. Levenson," $\chi^{(2)}$ semiconductor photonic crystals," J. Opt. Soc. Am. B 19, 2094-2101 (2002).

5. A. V. Andreev, A. V. Balakin, A. B. Kozlov, I. A. Ozheredov, I. R. Prudnikov, A. P. Shkurinov, P. Masselin, and G. Mouret, "Four-wave mixing in one-dimensional photonic crystals: inhomogeneous-wave excitation,” J. Opt. Soc. Am. B 19, 1865-1872 (2002).

6. G. J. Schneider and G. H. Watson, "Nonlinear optical spectroscopy in one-dimensional photonic crystals," Appl. Phys. Lett. 83, 5350-5352 (2003).

7. A. D. Bristow, J.-P. R. Wells, W. H. Fan, A. M. Fox, M. S. Skolnick, D. M. Whittaker, A. Tahraoui, T. F. Krauss, and J. S. Roberts, "Ultrafast nonlinear response of AlGaAs two-dimensional photonic crystal waveguides," Appl. Phys. Lett. 83, 851-853 (2003).

8. P. Xie and Z. Q. Zhang, "Optical phase conjugation in third-order nonlinear photonic crystals," Phys. Rev. A 69, 053806-5 (2004).

9. M. Scalora, J. P. Dowling, C. M. Bowden, and M. J. Bloemer, "Optical limiting and switching of ultrashort pulses in nonlinear photonic band gap materials," Phys. Rev. Lett. 73, 1368-1371 (1994)

10. S. John and T. Quang, "Optical bistability and phase transitions in a doped photonic band-gap material," Phys. Rev. A 54, 4479-4488 (1996).

11. R. Frey, P. Delaye, and G. Roosen. "Nonlinéarités optiques du troisième ordre dans les cristaux photoniques," in $L a$ Nanophotonique, C. Delalande, A. Levenson, and $\mathrm{H}$. Rigneault, eds. (Hermès-Lavoisier, to be published).

12. V. Berger, "Nonlinear photonic crystals," Phys. Rev. Lett. 81, 4136-4139 (1998).

13. M. Centini, G. D’Aguanno, L. Sciscione, C. Sibilia, M. Bertolotti, M. Scalora, and M. J. Bloemer, "Non-phasematched enhancement of second-harmonic generation in 
multilayer nonlinear structures with internal reflections," Opt. Lett. 29, 1924-1926 (2004).

14. Y. R. Shen, The Principles of Nonlinear Optics (Wiley, 1984).

15. J. Danckaert, K. Fobelets, I. Veretennicoff, G. Vitrant, and R. Reinisch "Dispersive optical bistability in stratified structures," Phys. Rev. B 44, 8214-8225 (1991).

16. C. Flytzanis, "Theory of nonlinear optical susceptibilities," in Quantum Electronics, C. L. Tang, ed. (Academic, 1975), Vol. I, part A.

17. A R. W. Boyd, Nonlinear Optics (Academic, 1992).

18. J. M. Bendickson, J. P. Dowling, and M. Scalora, "Analytic expressions for the electromagnetic mode density in finite, one-dimensional, photonic band-gap structures," Phys. Rev. E 53, 4107-4121 (1996).

19. F. Abelès, "Sur la propagation des ondes électromagnétiques dans les milieux stratifiés," Ann. Phys. (Paris) 3, 505 (1948).

20. P. Yeh, Optical Waves in Layered Media (Wiley, 1988).

21. L. Razzari, D. Trager, M. Astic, P. Delaye, R. Frey, G. Roosen, and R. André. "Kerr and four-wave mixing spectroscopy at the band edge of one-dimensional photonic crystals," Appl. Phys. Lett. 86, 231106(2005).

22. R. André, Laboratoire de Spectrométrie Physique, B. P. 87, 38402 Saint Martin d'Hères Cedex, France (private communication, 2003). 